\title{
An Improved Iterative Method for Solving the Discrete Algebraic Riccati Equation
}

\author{
Li Wang \\ School of Mathematics and Computational Science, Hunan University of Science and Technology, Hunan 411201, China \\ Correspondence should be addressed to Li Wang; wanglileigh@163.com
}

Received 24 March 2020; Accepted 21 April 2020; Published 20 May 2020

Guest Editor: Hou-Sheng Su

Copyright (C) $2020 \mathrm{Li}$ Wang. This is an open access article distributed under the Creative Commons Attribution License, which permits unrestricted use, distribution, and reproduction in any medium, provided the original work is properly cited.

The discrete algebraic Riccati equation has wide applications, especially in networked systems and optimal control systems. In this paper, according to the damped Newton method, two iterative algorithms with a stepsize parameter is proposed to solve the discrete algebraic Riccati equation, one of which is an extension of Algorithm (4.1) in Dai and Bai (2011). A numerical example demonstrates the convergence effect of the presented algorithm.

\section{Introduction and Preliminaries}

The discrete algebraic Riccati equation plays an important part in engineering, such as optimal control systems [1], modified filtering [2, 3], and networked systems [4-7]. Consider the following discrete-time linear system:

$$
x(k+1)=A x(k)+B u(k),
$$

where $x(k) \in R^{n}$ is the state variable, $u(k) \in R^{r}$ is the input variable, $B \in R^{n \times r}$ is the input matrix, and $A \in R^{n \times n}$ is the system matrix and is always invertible [8]. The optimal state feedback controller of (1) is

$$
u^{*}(k)=-\left(G+B^{T} P B\right)^{-1} B^{T} P A x(k),
$$

which minimizes the quadratic performance index of (1) and is closely related to the discrete algebraic Riccati equation (DARE):

$$
P=A^{T} P A-A^{T} P B\left(G+B^{T} P B\right)^{-1} B^{T} P A+Q,
$$

where $Q \in R^{n \times n}$ is semipositive definite, $G \in R^{r \times r}$ is positive definite, and $P \in R^{n \times n}$ is the positive definite solution of the DARE (3). Let $R=B G^{-1} B^{T} \geq 0$. According to the matrix identity,

$$
\left(X^{-1}+Y Z\right)^{-1}=X-X Y(I+Z X Y)^{-1} Z X,
$$

equation (3) can be transformed into

$$
P=A^{T}\left(P^{-1}+R\right)^{-1} A+Q .
$$

Due to the wide applications of the DARE, many works have been proposed to discuss the DARE. Various bounds and solutions about the DARE have been provided, such as upper and lower solution bounds [9-14], bounds about sum and product of eigenvalues $[15,16]$, determinant of the solution [17], and the existence of the solution [18-21]. However, in an optimal control system, we often need to compute the solution of the DARE to find the optimal state feedback controller which minimizes the quadratic performance index. It is very difficult to solve the DARE, especially when the dimensions of the coefficient matrices are high. So, many researchers provide a lot of iterative methods to solve this equation. Komaroff present a fixed-point iterative algorithm that needs to compute twice matrix inversion at each step [22]. By Newton's method, Guo derived the maximal symmetric solution of the DARE in [23]. The structure-preserving doubling algorithms are discussed in [24-27]. The Schur method is adopted to solve algebraic Riccati equations [28]. Recently, Dai and Bai propose an iterative algorithm that partially avoids computing the matrix inversions by making use of the Schulz iteration [29]. 
In Section 2, we propose two iterative algorithms with a stepsize parameter to solve the DARE by the damped Newton method. One of the iterative algorithms is an extension of Algorithm 4.1 in [29]. Numerical example is given in Section 3 to demonstrate the convergence effect of our algorithms.

We first introduce some symbol conventions. $R$ denotes the real number field. $R^{n \times m}$ denotes the set of $n \times m$ real matrices. For $X=\left(x_{i j}\right) \in R^{n \times n}$, let $X^{T}, X^{-1},\|X\|$, and $\lambda_{\text {min }}(X)$ denote the transpose, inverse, spectral norm, and the minimal eigenvalue of the matrix $X$, respectively. The inequality $X>(\geq) 0$ means $X$ is a symmetric positive (semi-) definite matrix; and the inequality $X>(\geq) Y$ means $X-Y$ is a symmetric positive (semi-) definite matrix. The identity matrix with appropriate dimensions is represented by $I$.

Lemma 1 (see [30]). If $A, B \in R^{n \times n}$ are symmetric positive definite matrices, then

$$
A \geq B, \quad \text { if and only if } \quad B^{-1} \geq A^{-1} .
$$

Lemma 2 (see [31]). Let $C$ and $P$ be Hermitian matrices of the same order and let $P>0$. Then,

$$
C P C+P^{-1} \geq 2 C \text {. }
$$

Lemma 3 (see [32]). Let Sand T be symmetric positive definite matrices. Then,

$$
\|S\| \geq\|T\|, \quad \text { if } \quad S \geq T \geq 0 .
$$

\section{Improved Iterative Algorithms for Solving the DARE}

To find the positive definite solution of the DARE (5), Dai and Bai, in [29], proposed an algorithm that partially avoids computing the matrix inversion as follows.

Algorithm 1 (see [29]). Take $Y_{0}=\left(Q^{-1}+R\right)^{-1}$. For $k=0,1,2, \cdots$, compute

$$
\left\{\begin{array}{l}
P_{k+1}=A^{T} Y_{k} A+Q \\
Y_{k+1}=Y_{k}\left(2 I-\left(P_{k+1}^{-1}+R\right) Y_{k}\right)
\end{array}\right.
$$

In this section, we propose two iterative algorithms to solve the DARE (5), which are motivated by the damped Newton method [33] and the methods in [34, 35]. Let us recall the damped Newton method to find the root of $F(Z)=Z^{-1}-B$ :

$$
Z_{k+1}=Z_{k}+t Z_{k}\left(I-B Z_{k}\right)=(1+t) Z_{k}-t Z_{k} B Z_{k},
$$

where $t>0$ is a stepsize parameter. If the initial matrix is near the solution of the problem, the unit stepsize $t=1$ can be accepted in the local Newton method. However, it is not suitable to choose $t=1$ if the initial matrix is far from the solution of the problem [33].

The DARE (5) can be translated into $F(P)=0$, where

$$
F(P)=\left[A^{-T}(P-Q) A^{-1}\right]^{-1}-\left(P^{-1}+R\right) .
$$

Let $Z=A^{-T}(P-Q) A^{-1}$ and $B=P^{-1}+R$. Then, to find the root of $F(P)$ is equivalent to find the root of $F(Z)$, we can solve the DARE (5) by constructing an iterative scheme. According to (10), we present the following iterative algorithms for the DARE (5).

Algorithm 2

Step 1: set $P_{0}=Q, Y_{0}=\left(Q^{-1}+R\right)^{-1}$ and $t>0$.

Step 2: compute

$$
\left\{\begin{array}{l}
Y_{k+1}=(1-t) Y_{k}+t\left[2 Y_{k}-Y_{k}\left(P_{k}^{-1}+R\right) Y_{k}\right] \\
P_{k+1}=A^{T} Y_{k+1} A+Q, \quad k=0,1,2, \cdots
\end{array}\right.
$$

Algorithm 3

Step 1: set $Y_{0}=\left(Q^{-1}+R\right)^{-1}, P_{0}=Q$ and $t>0$.

Step 2: compute

$$
\left\{\begin{array}{l}
P_{k+1}=A^{T} Y_{k} A+Q \\
Y_{k+1}=(1-t) Y_{k}+t\left[2 Y_{k}-Y_{k}\left(P_{k}^{-1}+R\right) Y_{k}\right], \quad k=0,1,2, \cdots
\end{array}\right.
$$

About Algorithms 2 and 3, we have the following results.

Theorem 1. Let $P_{-}$be the positive definite solution of the $D A R E$ (5) and $Q>0$. The iterative sequences $\left\{P_{k}\right\}$ and $\left\{Y_{k}\right\}$ are generated by Algorithm 2 with $t \in(0,1]$; then,

$$
\begin{aligned}
& P_{0} \leq P_{1} \leq P_{2} \leq \cdots, \quad \lim _{k \rightarrow \infty} P_{k}=P_{-} ; \\
& Y_{0} \leq Y_{1} \leq Y_{2} \leq \cdots, \quad \lim _{k \rightarrow \infty} Y_{k}=\left(P_{-}^{-1}+R\right)^{-1} .
\end{aligned}
$$

Proof. We first prove $P_{k}$ and $Y_{k}$ are monotone increasing by induction. Since $P_{-}$is positive definite solution of DARE (5), then

$$
P_{-}=A^{T}\left(P_{-}^{-1}+R\right)^{-1} A+Q
$$

Thus, $P_{-} \geq Q$.

(i) Since 


$$
\begin{aligned}
Y_{1} & =(1-t) Y_{0}+t\left[2 Y_{0}-Y_{0}\left(P_{0}^{-1}+R\right) Y_{0}\right] \\
& =(1-t) Y_{0}+t\left[2 Y_{0}-Y_{0}\left(Q^{-1}+R\right) Y_{0}\right] \\
& =(1-t) Y_{0}+t\left[2 Y_{0}-Y_{0}\right] \\
& =Y_{0},
\end{aligned}
$$

then by Lemma 1 , we obtain

$$
\begin{aligned}
P_{1} & =A^{T} Y_{1} A+Q=A^{T} Y_{0} A+Q \\
& =A^{T}\left(Q^{-1}+R\right)^{-1} A+Q, \\
& \leq A^{T}\left(P_{-}^{-1}+R\right)^{-1} A+Q \\
& =P_{-} .
\end{aligned}
$$

From (17), we also obtain $P_{1} \geq Q=P_{0}$; then,

$P_{-} \geq P_{1} \geq P_{0}$,

$Y_{0}=Y_{1}=\left(Q^{-1}+R\right)^{-1} \leq\left(P_{1}^{-1}+R\right)^{-1} \leq\left(P_{-}^{-1}+R\right)^{-1}$.

By Lemma 2 and (20), we have

$$
\begin{aligned}
Y_{2} & =(1-t) Y_{1}+t\left[2 Y_{1}-Y_{1}\left(P_{1}^{-1}+R\right) Y_{1}\right] \\
& \leq(1-t) Y_{1}+t\left(P_{1}^{-1}+R\right)^{-1} \\
& \leq(1-t)\left(P_{1}^{-1}+R\right)^{-1}+t\left(P_{1}^{-1}+R\right)^{-1} \\
& =\left(P_{1}^{-1}+R\right)^{-1} \leq\left(P_{-}^{-1}+R\right)^{-1} .
\end{aligned}
$$

By (20) and Lemma 1, we obtain

$$
\begin{aligned}
Y_{2} & =(1-t) Y_{1}+t\left[2 Y_{1}-Y_{1}\left(P_{1}^{-1}+R\right) Y_{1}\right] \\
& \geq(1-t) Y_{1}+t\left[2 Y_{1}-Y_{1}\left(Q^{-1}+R\right) Y_{1}\right] \\
& =(1-t) Y_{1}+t\left[2 Y_{1}-Y_{1}\right] \\
& =Y_{1},
\end{aligned}
$$

thereby,

$$
\begin{aligned}
P_{2} & =A^{T} Y_{2} A+Q \\
& \geq A^{T} Y_{1} A+Q=P_{1} .
\end{aligned}
$$

By (21), we obtain

$$
\begin{aligned}
P_{2} & =A^{T} Y_{2} A+Q \\
& \leq A^{T}\left(P_{1}^{-1}+R\right)^{-1} A+Q \\
& \leq A^{T}\left(P_{-}^{-1}+R\right)^{-1} A+Q=P_{-} .
\end{aligned}
$$

Thus, from the above-mentioned proof, we have

$$
\begin{aligned}
P_{0} & \leq P_{1} \leq P_{2} \leq P_{-}, \quad Y_{0}=Y_{1} \\
& \leq Y_{2} \leq\left(P_{1}^{-1}+R\right)^{-1} \leq\left(P_{-}^{-1}+R\right)^{-1} .
\end{aligned}
$$

(ii) Assume that

$$
\begin{aligned}
P_{i-1} & \leq P_{i} \leq \cdots \leq P_{-}, \quad Y_{i-1} \leq Y_{i} \leq\left(P_{i-1}^{-1}+R\right)^{-1} \\
& \leq\left(P_{-}^{-1}+R\right)^{-1}, \quad i=1,2, \cdots, k .
\end{aligned}
$$
then,

From (26), we get $Y_{k} \leq\left(P_{k-1}^{-1}+R\right)^{-1} \leq\left(P_{k}^{-1}+R\right)^{-1}$;

$$
Y_{k}^{-1} \geq P_{k}^{-1}+R
$$

Thus,

$$
\begin{aligned}
Y_{k+1} & =(1-t) Y_{k}+t\left[2 Y_{k}-Y_{k}\left(P_{k}^{-1}+R\right) Y_{k}\right] \\
& \geq(1-t) Y_{k}+t\left[2 Y_{k}-Y_{k} Y_{k}^{-1} Y_{k}\right] \\
& \geq(1-t) Y_{k}+t Y_{k}=Y_{k}, \\
Y_{k+1} & =(1-t) Y_{k}+t\left[2 Y_{k}-Y_{k}\left(P_{k}^{-1}+R\right) Y_{k}\right] \\
& \leq(1-t) Y_{k}+t\left(P_{k}^{-1}+R\right)^{-1} \\
& \leq(1-t)\left(P_{k}^{-1}+R\right)^{-1}+t\left(P_{k}^{-1}+R\right)^{-1} \\
& \leq\left(P_{k}^{-1}+R\right)^{-1} \leq\left(P_{-}^{-1}+R\right)^{-1} .
\end{aligned}
$$

By (28) and (29), we have

$$
\begin{gathered}
P_{k+1}=A^{T} Y_{k+1} A+Q \\
\geq A^{T} Y_{k} A+Q=P_{k}, \\
P_{k+1}=A^{T} Y_{k+1} A+Q \\
\leq A^{T}\left(P_{-}^{-1}+R\right)^{-1} A+Q=P_{-} .
\end{gathered}
$$

So, we obtain

$$
\begin{aligned}
P_{k} & \leq P_{k+1} \leq P_{-}, \quad Y_{k} \leq Y_{k+1} \leq\left(P_{k}^{-1}+R\right)^{-1} \\
& \leq\left(P_{-}^{-1}+R\right)^{-1}, k=0,1,2, \cdots
\end{aligned}
$$

Thus, the proof of induction is completed. Moreover, as $P_{k}$ and $Y_{k}$ are monotone increasing and they are bounded, then $\lim P_{k}$ and $\lim Y_{k}$ exist. Taking limits in Algorithm $^{k \longrightarrow \infty_{2}} \quad$ gives ${ }_{k}^{k \rightarrow \infty} \lim _{k \rightarrow \infty} Y_{k}=\left(P_{-}^{-1}+R\right)^{-1}$ and $\lim _{k \longrightarrow \infty} P_{k}=P_{-}$. 
Theorem 2. Let $P_{-}$be the positive definite solution of the $D A R E$ (5). After $k$ steps of iteration for Algorithm 2, we have $\left\|I-Y_{k}\left(P_{k}^{-1}+R\right)\right\|<\varepsilon$; then,

$$
\left\|A^{T}\left(P_{k}^{-1}+R\right)^{-1} A+Q-P_{k}\right\| \leq \varepsilon\left\|P_{-}-Q\right\| .
$$

Proof. According to (15), we have

$$
A^{T}\left(P_{-}^{-1}+R\right)^{-1} A=P_{-}-Q .
$$

Then, by Algorithm 2, Lemma 3, and (34), we obtain

$$
\begin{aligned}
\left\|A^{T}\left(P_{k}^{-1}+R\right)^{-1} A+Q-P_{k}\right\| & =\left\|A^{T}\left(P_{k}^{-1}+R\right)^{-1} A-A^{T} Y_{k} A\right\| \\
& =\left\|A^{T}\left[\left(P_{k}^{-1}+R\right)^{-1}-Y_{k}\right] A\right\| \\
& =\left\|A^{T}\left[I-Y_{k}\left(P_{k}^{-1}+R\right)\right]\left(P_{k}^{-1}+R\right)^{-1} A\right\| \\
& \leq\left\|I-Y_{k}\left(P_{k}^{-1}+R\right)\right\| \cdot\left\|A^{T}\left(P_{k}^{-1}+R\right)^{-1} A\right\| \\
& \leq\left\|I-Y_{k}\left(P_{k}^{-1}+R\right)\right\| \cdot\left\|A^{T}\left(P_{-}^{-1}+R\right)^{-1} A\right\| \\
& =\left\|I-Y_{k}\left(P_{k}^{-1}+R\right)\right\| \cdot\left\|P_{-}-Q\right\| \\
& \leq \varepsilon\left\|P_{-}-Q\right\|
\end{aligned}
$$

because of $P_{k} \leq P_{-}$.

As the proof method is similar to Theorem 1, we list the monotonicity and convergence of Algorithm 3 without proof.

Theorem 3. Let $P_{-}$be the positive definite solution of the $D A R E$ (5) and $Q>0$. The iterative sequences $\left\{P_{k}\right\}$ and $\left\{Y_{k}\right\}$ are generated by Algorithm 3 with $t \in 0,1]$ and start from $Y_{0}=\left(Q^{-1}+R\right)^{-1}$ and $P_{0}=Q$; then, $P_{k}$ is monotone increasing and converges to $P_{-}$, and $Y_{k}$ is monotone increasing and converges to $\left(P_{-}^{-1}+R\right)^{-1}$.

Remark 1. For Algorithms 2 and 3, we find the steps of iteration for Algorithm 2 are less than Algorithm 3 and the convergence speed of the Algorithm 2 is faster than Algorithm 3 from the numerical examples. Therefore, in the following example, we only discuss the superiority and effectiveness of Algorithm 2.

\section{Numerical Examples}

In this section, we present the following numerical example to show the effectiveness of our results. We also discuss the performance of Algorithm 2 with different $t$ values. The whole process is carried out on Matlab 7.1 and the precision is $10^{-8}$.
Example 1. Consider the discrete system (1) with

$$
\begin{aligned}
A & =\left(\begin{array}{cccc}
2.27 & 0.13 & 0.12 & 0.1 \\
-0.13 & 2.34 & 0.12 & 0.05 \\
0.11 & -0.17 & 1.9 & 0.03 \\
0.01 & 0.07 & 0.02 & 1.1
\end{array}\right), \\
B & =\left(\begin{array}{cccc}
1.15 & 0 & 0.01 & 0 \\
0 & 0.8 & 0 & 0 \\
0 & 0.04 & 0.9 & 0 \\
0.02 & 0 & 0 & 1.8
\end{array}\right) \\
Q & =\left(\begin{array}{cccc}
0.12 & 0 & 0.1 & 0 \\
0 & 2.2 & 0 & 0 \\
0.1 & 0 & 1.4 & 0 \\
0 & 0 & 0 & 0.7
\end{array}\right) \\
G & =I .
\end{aligned}
$$

In [29], Dai and Bai choose the starting matrix $Y_{0}=\left(Q^{-1}+R\right)^{-1}$. After 17 steps of iteration, the required precision is derived, and the residual $\| A^{T}\left(P^{-1}+R\right)^{-1} A+$ $Q-P \|$ is $2.0754 e-009$.

For Algorithm 2, we choose $P_{0}=Q, Y_{0}=\left(Q^{-1}+R\right)^{-1}$ and give the steps of iteration and the residual as Table 1 with 
TABLE 1: Numerical results.

\begin{tabular}{lccccc}
\hline$t$ & Iterations & Residual & $t$ & Iterations & Residual \\
\hline 0.6 & 35 & $9.0186 e-009$ & $\mathbf{1 . 2}$ & $\mathbf{1 0}$ & $5.6438 e-009$ \\
0.8 & 24 & $5.4562 e-009$ & 1.3 & 12 & $9.2094 e-009$ \\
0.9 & 20 & $4.7874 e-009$ & 1.5 & 18 & $9.4721 e-009$ \\
$\mathbf{1}$ & $\mathbf{1 7}$ & $2.0754 e-009$ & 1.8 & 40 & $8.6619 e-009$ \\
1.1 & 14 & $1.0160 e-009$ & 2.0 & 104 & $9.1141 e-009$ \\
\hline
\end{tabular}

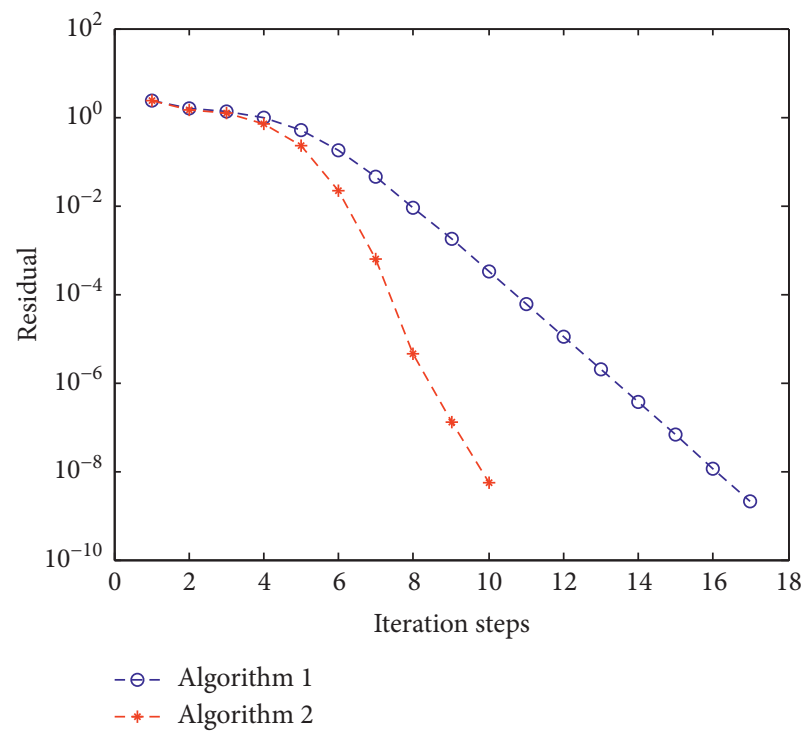

FIgURE 1: The relationship between iteration step and residual.

a different parameter $t$ when the process is stopped under the required precision. When $t$ is near 1 , we find that the steps of iteration are less than [29]. Especially, when $t=1.2$, it only needs 10 steps for Algorithm 2 to converge to the iterative solution:

$$
P_{10}=\left(\begin{array}{cccc}
3.3299 & -0.3120 & 0.5202 & 0.1433 \\
-0.3120 & 9.6394 & -0.1292 & 0.1904 \\
0.5202 & -0.1292 & 4.9731 & 0.0820 \\
0.1433 & 0.1904 & 0.0820 & 0.9962
\end{array}\right)
$$

with the residual $\left\|A^{T}\left(P^{-1}+R\right)^{-1} A+Q-P\right\|=5.6438 e-$ 009, and Algorithm 2 has faster convergence speed than Algorithm 1 from Figure 1. Moreover, from Table 1, we see that Algorithm 2 is more efficient when $t>1$. Although we only prove the convergence of Algorithm 2 when $t \in 0,1]$, in this paper, Algorithm 2 works well in practical computation when $t>1$.

\section{Data Availability}

All data generated or analyzed during this study are included within this article.

\section{Conflicts of Interest}

The authors declare that they have no conflicts of interest.

\section{Acknowledgments}

The work was supported in part by the National Natural Science Foundation for Youths of China (11801164), National Natural Science Foundation of China (11971413), Key Project of National Natural Science Foundation of China (91430213), General Project of Hunan Provincial Natural Science Foundation of China (2015JJ2134), and the General Project of Hunan Provincial Education Department of China (15C1320).

\section{References}

[1] H. Kwakernaak and R. Sivan, Linear Optimal Control Systems, Wiley-Interscience, New York, NY, USA, 1972.

[2] L. Wu and D. W. C. Ho, "Fuzzy filter design for Ito stochastic systems with application to sensor fault detection," IEEE Transactions on Fuzzy Systems, vol. 17, no. 1, pp. 233-242, 2009.

[3] X. Su, P. Shi, L. Wu, and Y. D. Song, "A novel approach to filter design for TCS fuzzy discrete-time systems with timevarying delay," IEEE Transactions on Fuzzy Systems, vol. 20, no. 6, pp. 1114-1129, 2012.

[4] W. N. Anderson, T. D. Morley, and G. E. Trapp, "Ladder networks, fixpoints, and the geometric mean," Circuits, Systems and Signal Processing, vol. 2, no. 3, pp. 259-268, 1983.

[5] M. Z. Q. Chen, L. Zhang, H. Su, and G. Chen, "Stabilizing solution and parameter dependence of modified algebraic Riccati equation with application to discrete-time network synchronization," IEEE Transactions on Automatic Control, vol. 61, no. 1, pp. 228-233, 2016.

[6] H. S. Su, H. Wu, and J. Lam, "Positive edge-consensus for nodal networks via output feedback," IEEE Transactions on Automatic Control, vol. 64, no. 3, pp. 1224-1249, 2019.

[7] M. Lan and S. Chand, "Solving linear quadratic discrete-time optimal controls usingneural networks," in Proceedings of the IEEE Conference on Decision \& Control, IEEE, Honolulu, HI, USA, December 1990.

[8] R. A. Kennedy, "Linear system theory," Automatica, vol. 30, no. 11, pp. 1811-1813, 1994.

[9] C.-H. Lee, "Matrix bounds of the solutions of the continuous and discrete Riccati equations-a unified approach," International Journal of Control, vol. 76, no. 6, pp. 635-642, 2003.

[10] H. H. Choi, "Upper matrix bounds for the discrete algebraic Riccati matrix equation," IEEE Transactions on Automatic Control, vol. 46, no. 3, pp. 504-508, 2001.

[11] R. Davies, P. Shi, and R. Wiltshire, "New upper solution bounds of the discrete algebraic Riccati matrix equation," Journal of Computational and Applied Mathematics, vol. 213, no. 2, pp. 307-315, 2008.

[12] J. Liu, L. Wang, and J. Zhang, “The solution bounds and fixed point iterative algorithm for the discrete coupled algebraic Riccati equation applied to automatic control," IMA Journal of Mathematical Control and Information, pp. 1-22, 2016.

[13] J. Liu, L. Wang, and J. Zhang, "New matrix bounds and iterative algorithms for the discrete coupled algebraic Riccati equation," International Journal of Control, vol. 90, no. 11, pp. 2326-2337, 2017.

[14] N. Komaroff, "Upper bounds for the solution of the discrete Riccati equation," IEEE Transactions on Automatic Control, vol. 37, no. 9, pp. 1370-1373, 1992.

[15] J. Zhang, J. Liu, and Y. Zha, "The improved eigenvalue bounds for the solution of the discrete algebraic Riccati equation," 
IMA Journal of Mathematical Control and Information, pp. 1-20, 2016.

[16] N. Komaroff and B. Shahian, "Lower summation bounds for the discrete Riccati and Lyapunov equations," IEEE Transactions on Automatic Control, vol. 37, no. 7, pp. 1078-1080, 1992.

[17] M. T. Tran and M. E. Sawan, "On the discrete Riccati matrix equation," SIAM Journal on Algebraic Discrete Methods, vol. 6, no. 1, pp. 107-108, 1985.

[18] J. Liu and J. Zhang, "The existence uniqueness and the fixed iterative algorithm of the solution for the discrete coupled algebraic Riccati equation," International Journal of Control, vol. 84, no. 8, pp. 1430-1441, 2011.

[19] R. Huang, J. Z. Liu, and L. Zhu, "Accurate solutions of diagonally dominant tridiagonal linear systems," BIT Numerical Mathematics, vol. 54, no. 3, pp. 711-727, 2014.

[20] Q. H. Liu, X. X. Li, and J. Yan, "On the large time behaviour of solutions for a class of time-dependent Hamilton-Jacobi equations," Science China Mathematics, vol. 59, no. 5, pp. 875-8890, 2016.

[21] Z.-H. He, "Some new results on a system of Sylvester-type quaternion matrix equations," Linear and Multilinear Algebra, pp. 1-23, 2019.

[22] N. Komaroff, "Iterative matrix bounds and computational solutions to the discrete algebraic Riccati equation," IEEE Transactions on Automatic Control, vol. 39, no. 8, pp. 16761678, 1994.

[23] C.-H. Guo, "Newton's method for discrete algebraic Riccati equations when the closed-loop matrix has eigenvalues on the unit circle," SIAM Journal on Matrix Analysis and Applications, vol. 20, no. 2, pp. 279-294, 1998.

[24] W.-W. Lin and S.-F. Xu, "Convergence analysis of structurepreserving doubling algorithms for riccati-type matrix equations," SIAM Journal on Matrix Analysis and Applications, vol. 28, no. 1, pp. 26-39, 2006.

[25] E. K.-W. Chu, H.-Y. Fan, W.-W. Lin, and C.-S. Wang, "Structure-preserving algorithms for periodic discrete-time algebraic Riccati equations," International Journal of Control, vol. 77, no. 8, pp. 767-788, 2004.

[26] L.-Z. Lu, W.-W. Lin, and C. E. M. Pearce, "An efficient algorithm for the discrete-time algebraic Riccati equation," IEEE Transactions on Automatic Control, vol. 44, no. 6, pp. 1216-1220, 1999.

[27] T.-M. Hwang, E. K.-W. Chu, and W.-W. Lin, “A generalized structure-preserving doubling algorithm for generalized discrete-time algebraic Riccati equations," International Journal of Control, vol. 78, no. 14, pp. 1063-1075, 2005.

[28] A. Laub, "A Schur method for solving algebraic Riccati equations," IEEE Transactions on Automatic Control, vol. 24, no. 6, pp. 913-921, 1979.

[29] H. Dai and Z. Z. Bai, "On eigenvalue bounds and iteration methods for discrete algebraic Riccati equations," Journal of Computational Mathematics, vol. 29, no. 3, pp. 341-366, 2011.

[30] R. A. Horn and C. R. Johnson, Matrix Analysis, Cambridge University Press, Cambridge, UK, 2012.

[31] X. Zhan, "Computing the extremal positive definite solutions of a matrix equation," SIAM Journal on Scientific Computing, vol. 17, no. 5, pp. 1167-1174, 1996.

[32] A. W. Marshall and I. Olkin, Inequalities: Theory of Majorization and its Applications, Academic Press, New York, NY, USA, 1979.

[33] W. Sun and Y. Yuan, Optimization Theory and Methods, Springer Science and Business Media, LLC, New York, NY, USA, 2006.
[34] M. Monsalve and M. Raydan, "A new inversion-free method for a rational matrix equation," Linear Algebra and Its Applications, vol. 433, no. 1, pp. 64-71, 2010.

[35] G. Schulz, "Iterative Berechung der reziproken Matrix," ZAMM-Zeitschrift für Angewandte Mathematik und Mechanik, vol. 13, no. 1, pp. 57-59, 1933. 